\title{
On the Cohen-Macaulay property for quadratic tangent cones
}

\author{
Dumitru I. Stamate * \\ Faculty of Mathematics and Computer Science \\ University of Bucharest \\ Str. Academiei 14, Bucharest 010014, Romania \\ and \\ Simion Stoilow Institute of Mathematics of the Romanian Academy \\ Research group of the project PN-II-RU-PD-2012-3-0656 \\ P.O.Box 1-764, Bucharest 014700, Romania \\ dumitru.stamate@fmi.unibuc.ro \\ Submitted: Dec 16, 2015; Accepted: Jul 17, 2016; Published: Aug 5, 2016 \\ Mathematics Subject Classifications: 13A30, 13H10, 16S37, \\ 13D40, 16S36, 13P10

\begin{abstract}
Let $H$ be an $n$-generated numerical semigroup such that its tangent cone $\operatorname{gr}_{\mathfrak{m}} K[H]$ is defined by quadratic relations. We show that if $n<5$ then $\operatorname{gr}_{\mathfrak{m}} K[H]$ is CohenMacaulay, and for $n=5$ we explicitly describe the semigroups $H$ such that $\operatorname{gr}_{\mathfrak{m}} K[H]$ is not Cohen-Macaulay. As an application we show that if the field $K$ is algebraically closed and of characteristic different from two, and $n \leqslant 5$ then $\operatorname{gr}_{\mathfrak{m}} K[H]$ is Koszul if and only if (possibly after a change of coordinates) its defining ideal has a quadratic Gröbner basis.
\end{abstract}

Keywords: numerical semigroup ring, tangent cone, Cohen-Macaulay, Koszul, $G$-quadratic, $h$-vector

\section{Introduction}

A numerical semigroup $H$ is a subset of $\mathbb{N}$ containing 0 and which is closed under addition such that the gcd of all elements in $H$ is 1 , or equivalently, such that $|\mathbb{N} \backslash H|<\infty$. We denote $\operatorname{Gen}(H)$ its unique minimal generating set. The embedding dimension of $H$ is defined $\operatorname{as} \operatorname{emb} \operatorname{dim}(H)=|\operatorname{Gen}(H)|$ and the multiplicity of $H$ is $e(H)=\min \operatorname{Gen}(H)$.

\footnotetext{
*Supported by a grant of the Romanian Ministry of Education, CNCS-UEFISCDI, project number PN-II-RU-PD-2012-3-0656.
} 
Let $K$ be an infinite field. The additive relations among the generators of $H$ are captured by the defining ideal $I_{H}$ of the semigroup ring $K[H]=K\left[t^{h}: h \in H\right] \subset K[t]$. Namely, if $\operatorname{Gen}(H)=\left\{a_{1}, \ldots, a_{n}\right\}$ and we let $S=K\left[x_{1}, \ldots, x_{n}\right]$, then $I_{H}=\operatorname{Ker} \phi$, where $\phi: S \rightarrow K[H]$ is the $K$-algebra map with $\phi\left(x_{i}\right)=t^{a_{i}}$, for $1 \leqslant i \leqslant n$.

Another algebra that is associated to $H$ is its tangent cone

$$
\operatorname{gr}_{\mathfrak{m}} K[H]=\oplus_{i \geqslant 0} \mathfrak{m}^{i} / \mathfrak{m}^{i+1},
$$

which is the associated graded ring of $K[H]$ with respect to the maximal ideal $\mathfrak{m}=\left(t^{h}\right.$ : $h \in H)$. The defining ideal of $\operatorname{gr}_{\mathfrak{m}} K[H]$ is $I_{H}^{*}$, the ideal of initial forms in $I_{H}$, see $[13$, $\S 15.10 .3]$ and $[15, \S 3.4]$.

It is a classical topic to study algebraic properties of $\operatorname{gr}_{\mathfrak{m}} K[H]$ like being CohenMacaulay or complete intersection (CI for short) in terms of the arithmetic of $H$, see [18], [22], [3], [10].

Algebras defined by quadratic relations occur naturally in algebraic geometry from varieties cut out by quadrics and they have been the initial framework for formulating several strong conjectures, e.g. what is currently known as the Eisenbud-Green-Harris conjecture introduced in [14, Section 4].

In recent work ([19]), J. Herzog and the author gave effective bounds for the multiplicity of a numerical semigroup $H$ such that $\operatorname{gr}_{\mathfrak{m}} K[H]$ is defined by quadrics. The motivation for the current paper came from the puzzling observation that all such numerical semigroups that we had obtained by blind computer search have the property that $\operatorname{gr}_{\mathfrak{m}} K[H]$ is Cohen-Macaulay.

Koszul algebras are an important class of quadratic algebras. Recall that a graded $K$-algebra $R=\oplus_{i \geqslant 0} R_{i}$ is called Koszul if $K$ has a graded $R$-linear resolution. $R$ is called $G$-quadratic if there exists a graded isomorphism $R \cong K\left[x_{1}, \ldots, x_{n}\right] / I$ where $I$ has a quadratic Gröbner basis with respect to some term order. It is well known that if $R$ is $G$-quadratic, then it is Koszul. We refer to [7] and [15] for proofs and related results on Koszul algebras.

For brevity, we say that a numerical semigroup $H$ is quadratic, Koszul, or $G$-quadratic, if $\operatorname{gr}_{\mathfrak{m}} K[H]$ has the respective property. Note that the quadratic property for $\operatorname{gr}_{\mathfrak{m}} K[H]$ depends on $H$ alone (see [21, Theorem 6.8]), however the other two might depend on the field $K$. It will be clear from the context, mainly in Section 2, which are our extra assumptions on $K$.

Let $H$ be a quadratic numerical semigroup. Using a criterion obtained independently by J. Herzog ([18]) and A. Garcia ([17]), and also our results from [19], we show in Proposition 5 that if emb $\operatorname{dim}(H)<5$, then $\operatorname{gr}_{\mathfrak{m}} K[H]$ is Cohen-Macaulay. It requires a bit more work to prove in Theorem 8 that if $\operatorname{emb} \operatorname{dim}(H)=5$, then $\operatorname{gr}_{\mathfrak{m}} K[H]$ is not Cohen-Macaulay precisely when $H$ is generated as

$$
\begin{gathered}
\left\langle 8,4 u^{\prime}, 4 u+2 u^{\prime}, 4 u^{\prime \prime}+2 u+u^{\prime}, 6 u+7 u^{\prime}+4 u^{\prime \prime}-8\right\rangle, \text { or } \\
\left\langle 8,4 u^{\prime}, 4 u+2 u^{\prime}, 4 u^{\prime \prime}+2 u+3 u^{\prime}, 6 u+9 u^{\prime}+4 u^{\prime \prime}-8\right\rangle,
\end{gathered}
$$

with $u, u^{\prime}, u^{\prime \prime}$ positive integers and $u^{\prime}>1$ is odd. Extending these exemples, in Proposition 7 for any $n>5$ we construct infinitely many $G$-quadratic numerical semigroups $H$ with 
$\operatorname{emb} \operatorname{dim}(H)=n$ and $\operatorname{gr}_{\mathfrak{m}} K[H]$ not Cohen-Macaulay. It would be interesting to figure out if such constructions cover all the cases when $\operatorname{gr}_{\mathfrak{m}} K[H]$ is quadratic and not CohenMacaulay.

In the terminology introduced by Rossi and Valla in [23], for $H$ in the above families the ideals $I_{H}$ provide first examples of 2-isomultiple ideals such that $\operatorname{gr}_{\mathfrak{m}} S / I_{H}$ is not Cohen-Macaulay. Their existence was also questioned in [23, Remark 2.3].

For a standard graded $K$-algebra $R=\oplus_{i \geqslant 0} R_{i}$ its $h$-polynomial $h(z)=\sum_{i \geqslant 0} h_{i} z^{i}$ is the numerator of the Hilbert series $H_{R}(z)=\sum_{i \geqslant 0} \operatorname{dim}_{K} R_{i} z^{i}$ when we write $H_{R}(z)=$ $h(z) /(1-z)^{d}$ with $h(1) \neq 0$. The $h$-vector of $R$ is the vector of coefficients $\left(h_{0}, h_{1}, \ldots\right)$ of the $h$-polynomial. Also, the (Hilbert-Samuel) multiplicity of $R$ is defined as $e(R)=h(1)$. It is known that for a numerical semigroup $H$ its multiplicity equals the multiplicity of the tangent cone $\operatorname{gr}_{\mathfrak{m}} K[H]$.

By work of Backelin, Conca and others, small values of $h_{2}$ imply the Koszul or the $G$-quadratic property of $R$, see [1], [4], [6], [9] and Lemma 10. If $R$ is Cohen-Macaulay and the field $K$ is infinite, we can mod out by a regular sequence of linear forms and the $h$-vector and the multiplicity are preserved. In case $H$ is a numerical semigroup and $R=\operatorname{gr}_{\mathfrak{m}} K[H]$ is Cohen-Macaulay, we may use $t^{e(H)}$ as a regular element.

As an application, in Section 2 we show that if $\operatorname{emb} \operatorname{dim}(H)<5$, then $H$ is quadratic if and only if it is $G$-quadratic. The first examples of quadratic and non-Koszul semigroups occur in embedding dimension 5 having multiplicity 9, e.g. $H=\langle 9,17,20,23,25\rangle$.

In a similar way, in [24] Roos and Sturmfels considered the Koszul property for quadratic projective monomial curves. Namely, given the relatively prime integers $0=$ $a_{1}<a_{1}<\cdots<a_{n}$, let $R=K\left[t_{1}^{a_{i}} t_{2}^{a_{n}-a_{i}}: 1 \leqslant i \leqslant n\right] \subset K\left[t_{1}, t_{2}\right]$. According to Table 1 in [24] obtained by a computer search, the first time when $R$ is quadratic and not Koszul is for $n=6$, and for $n=8$ occurs the first example where $R$ is Koszul and the associated toric ideal has no quadratic Gröbner basis.

Under the assumption that the field $K$ is algebraically closed and of characteristic $\neq 2$, we show in Theorem 12 that if $\operatorname{emb} \operatorname{dim}(H)=5$, then $H$ is Koszul if and only if it is $G$-quadratic. The proof works on the possible $h$-vectors of $\operatorname{gr}_{\mathrm{m}} K[H]$ when $H$ is quadratic, employing a result of Eisenbud, Green, and Harris in [14]. The assumptions on the field $K$ are due to Conca's results on the $G$-quadratic property for quadratic algebras with $h_{2} \leqslant 3$, see [4] and [6]. Screening the possible ideals $J=I_{H}^{*} \bmod x_{1}$ we found only two possible situations without a quadratic Gröbner basis, described in Remark 15. However, experimentally we found no quadratic semigroup $H$ producing such ideals.

We summarize our findings in Table 1 in Section 2 where we give a maximal list of 12 possible $h$-vectors of quadratic 5 -generated numerical semigroups. Note that experimentally we could not obtain the $h$-vectors $(1,4,3,1)$ and $(1,4,5)$. Nevertheless, we can conclude that if $H$ is quadratic and $\operatorname{emb} \operatorname{dim}(H) \leqslant 5$, the Hilbert function of $\operatorname{gr}_{\mathfrak{m}} K[H]$ is non-decreasing. This topic has been recently considered by D'Anna, Di Marca and Micale in [11] and by Oneto, Strazzanti and Tamone in [20]. 


\section{The Cohen-Macaulay condition}

In this section we study the Cohen-Macaulay property for the tangent cone of a quadratic numerical semigroup.

For further reference we first recall from our joint work with J. Herzog [19] some restrictions that we found on the multiplicity of a quadratic numerical semigroup.

Theorem 1. ([19, 1.1, 1.9, 1.12]) Let $H$ be a quadratic numerical semigroup minimally generated by $n>1$ elements and $K[H]$ its semigroup ring. Then

(i) $n \leqslant e(H) \leqslant 2^{n-1}$;

(ii) $e(H)=n \Longleftrightarrow I_{H}^{*}$ has a linear resolution;

(iii) $e(H)=2^{n-1} \Longleftrightarrow I_{H}^{*}$ is a CI ideal $\Longleftrightarrow I_{H}$ is a CI ideal;

(iv) if $\operatorname{gr}_{\mathfrak{m}} K[H]$ is Cohen-Macaulay and $e(H)<2^{n-1}$, then $e(H) \leqslant 2^{n-1}-2^{n-3}$.

Moreover, if we are in any of the situations from (ii), (iii) or if $\operatorname{gr}_{\mathfrak{m}} K[H]$ is CohenMacaulay and $e(H)=2^{n-1}-2^{n-3}$ then $H$ is $G$-quadratic, hence Koszul.

Remark 2. With notation as above, if $e(H)=n$, then $\operatorname{gr}_{\mathfrak{m}} K[H]$ has minimal multiplicity and by Sally's [25, Theorem 2] we get that $\operatorname{gr}_{\mathfrak{m}} K[H]$ is Cohen-Macaulay. We refer to the proof of Proposition 1.3 in [19] for related properties.

The following arithmetic result appeared in [19].

Lemma 3. ([19, Lemma 1.6]) Let $H$ be a numerical semigroup minimally generated by $a_{1}<a_{2}<\cdots<a_{n}$ with $n>1$. If $H$ is quadratic, then

(i) there exist $k, \ell \geqslant 2$ such that $a_{1} \mid a_{k}+a_{\ell}$.

(ii) $2 a_{i} \in\left\langle a_{1}, \ldots a_{i-1}, a_{i+1}, \ldots a_{n}\right\rangle$, for all $2 \leqslant i \leqslant n$.

For the numerical semigroup $H$ minimally generated by $a_{1}<\cdots<a_{n}$ we denote

$$
c_{i}=\min \left\{k>0: k a_{i} \in\left\langle\operatorname{Gen}(H) \backslash\left\{a_{i}\right\}\right\rangle\right\} \text {, for } i=1, \ldots, n .
$$

With this notation one has the following characterization proved independently by Herzog ([18]) and Garcia ([17]).

Proposition 4. (Herzog [18, pp.189-190], Garcia [17, Theorem 24]) The tangent cone $\operatorname{gr}_{\mathfrak{m}} K[H]$ is Cohen-Macaulay if and only if for all integers $0 \leqslant \nu_{i}<c_{i}$ and $2 \leqslant i \leqslant n$ such that

$$
\sum_{i=2}^{n} \nu_{i} a_{i} \in a_{1}+H
$$

there exist integers $\mu_{1}>0, \mu_{2} \geqslant 0, \ldots, \mu_{n} \geqslant 0$ such that

$$
\sum_{i=2}^{n} \nu_{i} a_{i}=\sum_{i=1}^{n} \mu_{i} a_{i} \quad \text { and } \quad \sum_{i=2}^{n} \nu_{i} \leqslant \sum_{i=1}^{n} \mu_{i} .
$$


If $H$ is quadratic, by Lemma 3(ii) we have $c_{i}=2$ for $i=2, \ldots, n$. This observation, together with Proposition 4 , gives the next result.

Proposition 5. If $H$ is a quadratic semigroup and $\operatorname{emb} \operatorname{dim}(H)<5$ then $\operatorname{gr}_{\mathfrak{m}} K[H]$ is Cohen-Macaulay.

Proof. If emb $\operatorname{dim}(H)=2$ then $H=\langle 2, \ell\rangle$ with $\ell>1$ odd. Hence $\operatorname{gr}_{\mathfrak{m}} K[H] \cong K\left[x_{1}, x_{2}\right] /\left(x_{2}^{2}\right)$ which is Cohen-Macaulay.

If $\operatorname{emb} \operatorname{dim}(H)=3$, by Theorem 1 (i) we have $3 \leqslant e(H) \leqslant 4$, and by (ii) and (iii) in loc.cit. $\operatorname{gr}_{\mathfrak{m}} K[H]$ is Cohen-Macaulay.

If $\operatorname{emb} \operatorname{dim}(H)=4$ let

$$
\nu_{2} a_{2}+\nu_{3} a_{3}+\nu_{4} a_{4}=\mu_{1} a_{1}+\mu_{2} a_{2}+\mu_{3} a_{3}+\mu_{4} a_{4}
$$

for some $\mu_{1}>0, \mu_{2}, \mu_{3}, \mu_{4} \geqslant 0$ and $\nu_{2}, \nu_{3}, \nu_{4} \in\{0,1\}$. It is enough to consider the case when not both of $\nu_{2}$ and $\mu_{2}$, of $\nu_{3}$ and $\mu_{3}$, and of $\nu_{4}$ and $\mu_{4}$ are positive. Note that since $\operatorname{emb} \operatorname{dim}(H)=4$ at least two of the $\nu_{i}^{\prime}$ 's need to be positive.

If $\nu_{2}=\nu_{3}=1$ and $\nu_{4}=0$ then in the equation $a_{2}+a_{3}=\mu_{1} a_{1}+\mu_{4} a_{4}$ we have either $\mu_{4}>0$, hence $\mu_{1}+\mu_{4} \geqslant 2=\nu_{2}+\nu_{3}$, or $\mu_{4}=0$, hence $a_{2}+a_{3}=\mu_{1} a_{1}$ with $\mu_{1}>2$. The cases $\nu_{3}=\nu_{4}=1, \nu_{2}=0$ and $\nu_{2}=\nu_{4}=1, \nu_{3}=0$ are treated similarly.

If $\nu_{2}=\nu_{3}=\nu_{4}=1$ then in the equation $a_{2}+a_{3}+a_{4}=\mu_{1} a_{1}$ we have $\mu_{1}>3$.

By Proposition 4 it follows that $\operatorname{gr}_{\mathfrak{m}} K[H]$ is Cohen-Macaulay.

Example 6. The statement of Proposition 5 is no longer true when emb $\operatorname{dim}(H)$ is at least 5 . We can check with Singular $([16])$ that for $H=\langle 8,12,13,18,35\rangle$ the ideal $I_{H}^{*}$ has a quadratic Gröbner basis with respect to revlex, however $\operatorname{gr}_{\mathfrak{m}} K[H]$ is not Cohen-Macaulay.

Indeed, the toric ideal $I_{H}$ is minimally generated by

$$
\begin{aligned}
I_{H}=\left(x_{3}^{2}-x_{1} x_{4}, x_{2} x_{4}^{2}-x_{3} x_{5},\right. & x_{2} x_{3} x_{4}-x_{1} x_{5}, x_{2}^{3}-x_{4}^{2}, \\
& \left.x_{1}^{3}-x_{2}^{2}, x_{1}^{2} x_{3} x_{4}-x_{2} x_{5}, x_{1}^{2} x_{4}^{3}-x_{5}^{2}, x_{1}^{2} x_{2}^{2} x_{3}-x_{4} x_{5}\right) .
\end{aligned}
$$

A revlex Gröbner basis for $I_{H}^{*}$ is given by

$$
I_{H}^{*}=\left(x_{5}^{2}, x_{4} x_{5}, x_{3} x_{5}, x_{2} x_{5}, x_{1} x_{5}, x_{4}^{2}, x_{3}^{2}-x_{1} x_{4}, x_{2}^{2}\right),
$$

and $\left(I_{H}^{*}: x_{5}\right)=\left(x_{1}, x_{2}, x_{3}, x_{4}, x_{5}\right)$, hence $\operatorname{depth}_{\mathfrak{m}} K[H]=0$.

This is not an isolated example. For any given embedding dimension $n>4$ we construct infinitely many $G$-quadratic numerical semigroups whose tangent cone is not Cohen-Macaulay. But first we recall a useful construction.

Let $L$ be a numerical semigroup, $\ell$ an odd integer in $L$ and $H=\langle 2 L, \ell\rangle$. By [19, Definition 2.2], the semigroup $H$ is called a quadratic gluing of $L$. It is proved in [19, Corollary 2.7] that $L$ and $H$ are quadratic, Koszul, respectively G-quadratic, at the same time. It is also known by Delorme's work [12] that if $L$ is a complete intersection (CI), then so is $H$. We refer to Section 2 in [19] for more details about the CI property for quadratic numerical semigroups. 
Proposition 7. Given $n \geqslant 3$ and the positive integers $u_{i}, i=1, \ldots, n+1$, where $u_{1} \geqslant 3$ is odd, let

$$
\begin{aligned}
a_{1} & =2^{n}, \\
a_{2} & =2^{n-1} u_{1}, \\
a_{3} & =2^{n-1} u_{2}+2^{n-2} u_{1}, \\
\cdots & \\
a_{n+1} & =2^{n-1} u_{n}+2^{n-2} u_{n-1}+\cdots+u_{1}, \\
a_{n+2} & =a_{2}+\cdots+a_{n+1}-a_{1} .
\end{aligned}
$$

The semigroup $H=\left\langle a_{1}, \ldots, a_{n+2}\right\rangle$ is a G-quadratic numerical semigroup of embedding dimension $n+2$ and $\operatorname{gr}_{\mathfrak{m}} K[H]$ is not Cohen-Macaulay.

Letting $n=3, u_{1}=u_{2}=3$ and $u_{3}=1$ in the construction above, we obtain the quadratic semigroup $H=\langle 8,12,18,13,35\rangle$ from Example 6. Note that in Proposition 7 the listed generators $a_{i}$ are not necessarily in increasing order, however we always have $a_{1}<a_{i}<a_{n+2}$ for $2 \leqslant i \leqslant n+1$.

Proof. Denote $H_{i}=\left\langle a_{1}, \ldots, a_{i}\right\rangle$ for $1 \leqslant i \leqslant n+2$. It is easy to see that $\operatorname{gcd}\left(a_{1}, \ldots, a_{i}\right)=$ $2^{n-i+1}$ for $i=1, \ldots, n+1$, hence $H_{n+1}$ and $H$ are numerical semigroups. From the defining relations we infer that

$$
2 a_{i+1}=2^{n} u_{i}+a_{i} \quad \text { for } 2 \leqslant i \leqslant n,
$$

hence the (not necessarily numerical) semigroups $H_{1}, \ldots, H_{n+1}$ are obtained by quadratic gluings, are CI and $G$-quadratic. Also, from the equations (1) we see that

$$
I_{H_{i}}=\left(x_{2}^{2}-x_{1}^{u_{1}}\right)+\left(x_{j+1}^{2}-x_{1}^{u_{j}} x_{j}: 2 \leqslant j<i\right) \quad \text { for } 2 \leqslant i \leqslant n+1 .
$$

Next we compute $I_{H}$ and $I_{H}^{*}$. We note that $a_{n+2}+a_{1}=a_{2}+\cdots+a_{n+1}$. For $2 \leqslant i \leqslant n+1$ using (1) repeatedly we get

$$
\begin{aligned}
a_{n+2}+a_{i}= & a_{2}+\cdots+a_{i-1}+2 a_{i}+\sum_{j=i+1}^{n+1} a_{j}-2^{n} \\
= & a_{2}+\cdots+a_{i-1}+\left(a_{i-1}+2^{n} u_{i-1}\right)+\sum_{j=i+1}^{n+1} a_{j}-2^{n} \\
= & a_{2}+\cdots+a_{i-2}+\left(a_{i-2}+2^{n} u_{i-2}\right)+2^{n} u_{i-1}+\sum_{j=i+1}^{n+1} a_{j}-2^{n} \\
& \cdots \\
= & 2^{n}\left(u_{i-1}+\cdots+u_{1}-1\right)+\sum_{j=i+1}^{n+1} a_{j} \\
= & \left(u_{i-1}+\cdots+u_{1}-1\right) a_{1}+\sum_{j=i+1}^{n+1} a_{j} .
\end{aligned}
$$


Arguing similarly we obtain

$$
2 a_{n+2}=\left(u_{1}+\cdots+u_{n}-2\right) a_{1}+\sum_{i=2}^{n} a_{i} .
$$

Each of these relations produces a binomial in $I_{H}$ :

$$
\begin{aligned}
f_{1} & =x_{1} x_{n+2}-\prod_{j=2}^{n+1} x_{j}, \\
f_{i} & =x_{i} x_{n+2}-x_{1}^{\left(\sum_{j=1}^{i-1} u_{j}\right)-1} \prod_{j=i+1}^{n+1} x_{j}, \text { for } 2 \leqslant i \leqslant n+1, \text { and } \\
f_{n+2} & =x_{n+2}^{2}-x_{1}^{\left(\sum_{j=1}^{n} u_{j}\right)-2} \prod_{j=2}^{n} x_{j} .
\end{aligned}
$$

By inspecting these relations we remark that we can always choose a generating set for $I_{H}$ consisting of binomials such that in each monomial in the support, different from $x_{n+2}^{2}$, the variable $x_{n+2}$ has degree at most one. Therefore, $I_{H}=\left(I_{H_{n+1}}, f_{1}, \ldots, f_{n+2}\right)$.

Since $n \geqslant 3$ and $u_{1} \geqslant 3$ it is easy to see that $f_{i}^{*}=x_{i} x_{n+2}$ for $i=1, \ldots, n+2$. Arguing as above we derive

$$
I_{H}^{*}=I_{H_{n+1}}^{*}+x_{n+2}\left(x_{1}, \ldots, x_{n+2}\right) .
$$

This gives $I_{H}^{*}: x_{n+2}=\left(x_{1}, \ldots, x_{n+2}\right)$ and depth $\operatorname{gr}_{\mathfrak{m}} K[H]=0$, hence $R=\operatorname{gr}_{\mathfrak{m}} K[H]$ is not Cohen-Macaulay.

Since $x_{n+2} R_{1}=0$, by Conca's [4, Lemma 4.(1)] we have that $R$ is $G$-quadratic if and only if

$$
R /\left(t^{a_{n+2}}\right) \cong K\left[x_{1}, \ldots, x_{n+2}\right] /\left(I_{H}^{*}, x_{n+2}\right) \cong K\left[x_{1}, \ldots, x_{n+1}\right] / I_{H_{n+1}}^{*} \cong \operatorname{gr}_{\mathfrak{m}} K\left[H_{n+1}\right]
$$

is $G$-quadratic, which is true since $H_{n+1}$ is a quadratic CI, see Theorem 1.

Our next goal is to identify the quadratic numerical semigroups $H$ of embedding dimension 5 and $\operatorname{gr}_{\mathfrak{m}} K[H]$ not Cohen-Macaulay.

Theorem 8. Let $H$ be a quadratic numerical semigroup with emb $\operatorname{dim}(H)=5$. Then $\operatorname{gr}_{\mathfrak{m}} K[H]$ is not Cohen-Macaulay if and only if $H$ is of any of the following forms:

(i) $H=\left\langle 8,4 u^{\prime}, 4 u+2 u^{\prime}, 4 u^{\prime \prime}+2 u+u^{\prime}, 6 u+7 u^{\prime}+4 u^{\prime \prime}-8\right\rangle$ with $u, u^{\prime}, u^{\prime \prime}$ positive integers and $u^{\prime}>1$ is odd, or

(ii) $H=\left\langle 8,4 u^{\prime}, 4 u+2 u^{\prime}, 4 u^{\prime \prime}+2 u+3 u^{\prime}, 6 u+9 u^{\prime}+4 u^{\prime \prime}-8\right\rangle$ with $u, u^{\prime}, u^{\prime \prime}$ positive integers and $u^{\prime}>1$ is odd.

Whenever $H$ is in any of these two families, it is also G-quadratic. 
Proof. We first assume $H$ is in any of the specified families and we show that $\operatorname{gr}_{\mathfrak{m}} K[H]$ is not Cohen-Macaulay. We label $a_{1}, \ldots, a_{5}$ the generators of $H$ in the given ordering.

For $(i)$ we are in the situation described in Proposition 7 for $n=3, u_{1}=u^{\prime}, u_{2}=u$ and $u_{3}=u^{\prime \prime}$, hence the conclusion follows.

For $(i i)$ we note that the semigroup $L=\left\langle a_{1}, a_{2}, a_{3}, a_{4}\right\rangle=\left\langle 2\left\langle 4,2 u^{\prime}, 2 u+u^{\prime}\right\rangle, a_{4}\right\rangle$ is obtained by a quadratic gluing since $a_{4}=u^{\prime \prime} \cdot 4+\left(2 u+u^{\prime}\right)+\left(2 u^{\prime}\right)$ is odd. By [19, Proposition 3.6] the semigroup $\left\langle 4,2 u^{\prime}, 2 u+u^{\prime}\right\rangle$ is a quadratic complete intersection, and the same holds for $L$ by Delorme's [12, Proposition 9] and by [19, Corollary 2.7].

It is straightforward to check that

$$
\begin{aligned}
a_{5}+a_{1} & =a_{2}+a_{3}+a_{4}, \\
a_{5}+a_{2} & =\left(u^{\prime}-1\right) a_{1}+a_{3}+a_{4}, \\
a_{5}+a_{3} & =\left(u+u^{\prime}-1\right) a_{1}+a_{4}, \\
a_{5}+a_{4} & =\left(u+u^{\prime \prime}-1\right) a_{1}+3 a_{2}, \\
2 a_{5} & =\left(u+2 u^{\prime}+u^{\prime \prime}-2\right) a_{1}+a_{3},
\end{aligned}
$$

hence $I_{H}^{*}: x_{5}=\left(x_{1}, \ldots, x_{5}\right)$ and $\operatorname{gr}_{\mathfrak{m}} K[H]$ is not Cohen-Macaulay. Arguing as in the proof of Proposition 7 we get that $H$ is $G$-quadratic.

The direct implication is proved separately in Section 3.

\section{Koszul and $G$-quadraticity}

As an application of Theorem 8, under some restrictions on the field $K$, we prove that if $H$ is a numerical semigroup and $\operatorname{emb} \operatorname{dim}(H) \leqslant 5$, then $H$ is Koszul if and only if it is $G$-quadratic. We wonder if this statement holds for arbitrary embedding dimension.

Let $R=\oplus_{i \geqslant 0} R_{i}$ be a standard graded $K$-algebra. A Koszul filtration for $R$ is a family $\mathcal{F}$ of ideals of $R$ generated by linear forms such that 0 and the maximal homogeneous ideal of $R$ belong to $\mathcal{F}$ and for every $I \in \mathcal{F}$ different from 0 , there exists $J \in \mathcal{F}$ such that $J \subset I, I / J$ is cyclic and $J: I \in \mathcal{F}$. A Koszul filtration that is totally ordered with respect to inclusion is called a Gröbner flag. It is known that if $R$ has a Koszul filtration, then it is Koszul. Also, by [5, Theorem 2.4], if $R$ has a Gröbner flag, then $R$ is $G$-quadratic. We refer to the original papers [8], [5] and to the recent survey [7] of Conca et al. for more properties.

For easier reference we group in the following lemma some known results about lifting Koszul-like properties modulo a linear form.

Lemma 9. Let $R$ be a standard graded $K$-algebra and $x$ a linear form that is regular on $R$. If $R /(x)$ has property $(\mathcal{P})$, then so does $R$, where $\mathcal{P}$ stands for Koszul, G-quadratic, admits a Koszul filtration, or a Gröbner flag.

Proof. The statements for Koszul and $G$-quadraticity are due to Backelin and Fröberg in [2, Lemma 2], respectively to Conca in [4, Lemma 4.(2)].

That any Gröbner flag may be lifted from $R /(x)$ to $R$ is proved in [5, Lemma 2.11.(a)]. Using the same idea one can produce a Koszul filtration for $R$ from a Koszul filtration of $R /(x)$. 
For $R$ as above its $h$-polyonomial is defined as the numerator $h(t)$ in the writing of the Hilbert series $H_{R}(t)=\Sigma_{i \geqslant 0} \operatorname{dim}_{K} R_{i} t^{i}=h(t) /(1-t)^{d}$ with $h(1) \neq 0$. If $h(t)=\Sigma_{i \geqslant 0} h_{i} t^{i}$, the sequence of coefficients $\left(h_{0}, h_{1}, \ldots\right)$ is called the $h$-vector of $R$.

It is clear that if $x$ is a linear form which is regular on $R$, the $h$-polynomial and the $h$-vector of $R$ and $R /(x)$ are the same. In this context, the Cohen-Macaulay property for $R$ facilitates the computation of the $h$-vector of $R$ by reduction to the artinian case.

For a quadratic (artinian) $K$-algebra the Koszul property or the existence of a quadratic Gröbner basis, or of a Gröbner flag may sometimes be deduced by inspecting the $h$-vector. We collect some results on this topic that we will use later on.

Lemma 10. Let $R=\oplus_{i \geqslant 0} R_{i}$ be a quadratic standard graded $K$-algebra.

(i) (Conca, Rossi, Valla, [5, Proposition 2.12]) If $H_{R}(t)=1+n t+t^{2}$ with $n>1$, then $R$ has a Gröbner flag.

(ii) (Backelin, [1, Theorem 4.8]) If $\operatorname{dim}_{K} R_{2} \leqslant 2$, then $R$ is Koszul.

Assume the field $K$ is algebraically closed and of characteristic $\neq 2$.

(iii) (Conca, [4, Theorem 1]) If $\operatorname{dim}_{K} R_{2} \leqslant 2$, then $R$ is $G$-quadratic if and only if it is not graded isomorphic to the $K$-algebra $K[x, y, z] /\left(x^{2}, x y, y^{2}-x z, y z\right)$ or its trivial fiber extentions.

(iv) (Conca, [6, Theorem 1.1]) If $R$ is artinian and $\operatorname{dim}_{K} R_{2}=3$, then $R$ is Koszul. Moreover, $R$ is G-quadratic if and only if it is not a trivial fiber extension of $K[x, y, z] / I$, where $I$ is a complete intersection of three quadrics not containing the square of a linear form.

It is now easier to prove the announced statement for the case when $\operatorname{emb} \operatorname{dim}(H)$ is less than 5 .

Theorem 11. Let $H$ be a numerical semigroup. If emb $\operatorname{dim}(H)<5$, then $H$ is quadratic if and only if $H$ is $G$-quadratic.

Proof. Denote $n=\operatorname{emb} \operatorname{dim}(H)$ and $R=\operatorname{gr}_{\mathfrak{m}} K[H]$. Assume $H$ is quadratic. If $n=2$ then $R$ is a hypersurface ring, and we are done. If $n=3$, then $e(H) \in\{3,4\}$ and the result follows from Theorem 1 .

Assume $n=4$ and then $R$ is Cohen-Macaulay by Proposition 5 . Denote $\bar{R}=R /\left(x_{1}\right)$. Using Theorem 1 we get that $e(H) \in\{4,5,6,8\}$. If $e(H) \neq 5$, then we are in one of the cases covered by Theorem 1 and the conclusion follows. If $e(H)=5$, then $\ell(\bar{R})=e(H)$ and $\bar{R}$ has the $h$-vector $(1,3,1)$. By Lemma $10(\mathrm{i})$ we obtain that $\bar{R}$ has a Gröbner flag which by Lemma 9 produces a Gröbner flag for $R$. Hence $R$ is $G$-quadratic.

For a 5-generated quadratic numerical semigroup there are more possible multiplicities for which the Koszul property does not follow easily from Theorem 1. Our analysis depends on the possible $h$-polynomial of $R=\operatorname{gr}_{\mathfrak{m}} K[H]$ when $H$ is quadratic. We combine the results listed in Lemma 10 and Theorem 8 with computer testing in Singular $([16])$ 
for the remaining situations. Before giving the main result, we describe our screening strategy performed on the computer.

Working under the assumption that $x_{1}$ is regular on $R$, we analysed the possible quadratic ideals $J=I_{H}^{*} \bmod \left(x_{1}\right)$ in $K\left[x_{2}, x_{3}, x_{4}, x_{5}\right]$ generated by monomials and binomials and subject to some restrictions due to our setup. Choose $\mathcal{G}$ any minimal generating set for $I_{H}$ consisting of binomials. If we denote $\mathcal{G}^{*}$ the collection of their initial forms, by [19, Lemma 1.5] $\mathcal{G}^{*}$ generates $I_{H}^{*}$ minimally. Since $x_{1}$ is regular on $R$, the set $\mathcal{H}$ obtained by letting $x_{1}=0$ in $\mathcal{G}^{*}$ is a minimal generating set for $J$ consisting of quadratic monomials and possibly of binomials.

Since the variables correspond to the generators of $H$ taken in increasing order, there is only a short list of possible binomials in $\mathcal{H}$ :

$$
\begin{gathered}
p_{0}=x_{2} x_{5}-x_{3} x_{4}, \quad p_{1}=x_{3}^{2}-x_{2} x_{4}, \quad p_{2}=x_{3}^{2}-x_{2} x_{5}, \\
p_{3}=x_{4}^{2}-x_{2} x_{5}, \quad p_{4}=x_{4}^{2}-x_{3} x_{5} .
\end{gathered}
$$

Clearly $p_{1}$ and $p_{2}$ can not occur at the same time in $\mathcal{H}$, otherwise $p_{1}-p_{2}=x_{2}\left(x_{4}-x_{5}\right) \in I_{H}$, hence $x_{4}-x_{5} \in I_{H}$, which is false. Similarly, $p_{3}$ and $p_{4}$ may not both occur $\mathcal{H}$. Hence at most 3 binomials may occur simultaneously in $\mathcal{H}$.

On the other hand, if $p_{0}$ and $p_{1}$ occur in $\mathcal{H}$, these occur in $I_{H}$, too. Hence $a_{2}+a_{5}=$ $a_{3}+a_{4}$ and $2 a_{3}=a_{2}+a_{4}$. Adding these equations we get $a_{3}+a_{5}=2 a_{4}$, therefore $p_{4} \in I_{H}$. We get that either $p_{4} \in \mathcal{H}$, or that the monomials in its support are in $\mathcal{H}$.

Arguing similarly we see that if any two of $p_{0}, p_{1}$ and $p_{4}$ occur in $\mathcal{H}$, then the remaining one is in $I_{H}$ and in $J$.

By Lemma 3 we see that $x_{2}^{2}, x_{5}^{2} \in \mathcal{H}$. Also, if $x_{3}^{2} \notin \mathcal{H}$ then either $p_{1}$ or $p_{2}$ is in $\mathcal{H}$. Similarly, if $x_{4}^{2} \notin \mathcal{H}$, then either $p_{3}$ or $p_{4}$ is in $\mathcal{H}$.

Theorem 12. Let $H$ be a numerical semigroup with $\operatorname{emb} \operatorname{dim}(H)=5$.

If the field $K$ is algebraically closed and of characteristic $\neq 2$, then $H$ is Koszul if and only if it is G-quadratic.

Proof. By Theorem 8, if $R=S / I_{H}^{*} \cong \operatorname{gr}_{\mathfrak{m}} K[H]$ is not Cohen-Macaulay then it is $G$ quadratic. So it is enough to consider the case when $x_{1}$ is a nonzero divisor on $R$. For $i=2, \ldots, 5$, there exist distinct polynomials in $I_{H}^{*}$ of the form $f_{i}=x_{i}^{2}-g_{i}$, where $g_{i}$ is either zero or a quadratic monomial which is not a pure power. Denoting by an overbar ${ }^{-}$ the image in $\bar{R}=R /\left(x_{1}\right)$ and $<$ the revlex term order induced by $x_{5}>x_{4}>\ldots$, we have $\operatorname{in}_{<}\left(\bar{f}_{i}\right)=x_{i}^{2}$ for $2 \leqslant i \leqslant 5$. Therefore the Hilbert series of the artinian graded algebras $\bar{R}$ and $K\left[x_{2}, \ldots, x_{5}\right] / \operatorname{in}_{<}(J)$ coincide and moreover $\operatorname{in}_{<}(J)$ contains the squares of all the variables.

In this situation, as noted by Eisenbud, Green and Harris in [14, Section 4], for any $m$, if $h_{m}=\operatorname{dim}_{K} \bar{R}_{m}$ has the binomial decomposition

$$
h_{m}=\left(\begin{array}{c}
b_{m} \\
m
\end{array}\right)+\left(\begin{array}{c}
b_{m-1} \\
m-1
\end{array}\right)+\cdots+\left(\begin{array}{c}
b_{1} \\
1
\end{array}\right)
$$

with $b_{m}>b_{m-1}>\cdots>b_{1} \geqslant 0$, then

$$
h_{m+1} \leqslant\left(\begin{array}{c}
b_{m} \\
m+1
\end{array}\right)+\left(\begin{array}{c}
b_{m-1} \\
m
\end{array}\right)+\cdots+\left(\begin{array}{c}
b_{1} \\
2
\end{array}\right) .
$$


The $h$-vector of $\bar{R}$ is $\left(1,4, h_{2}, h_{3}, h_{4}\right)$. By (2) we have $0 \leqslant h_{2} \leqslant\left(\begin{array}{l}4 \\ 2\end{array}\right)=6$. Our analysis depends on the possible values for $h_{2}$.

- If $h_{2}=0$, then $\ell(\bar{R})=e(H)=5$, and by Theorem $1, H$ is $G$-quadratic.

- If $h_{2}=1$, then by (2) we get $h_{3}=0$, hence the $h$-vector of $\bar{R}$ is $(1,4,1)$. By Lemma $10(\mathrm{i})$ and Lemma 9 we get that $\bar{R}$ and $R$ have a Gröbner flag, hence they are $G$-quadratic.

- If $h_{2}=2=\left(\begin{array}{l}2 \\ 2\end{array}\right)+\left(\begin{array}{l}1 \\ 1\end{array}\right)$, from (2) we deduce that $h_{3}=0$. Since $J$ is artinian and the field $K$ is algebraically closed of characteristic $\neq 2$, by Lemma 10(iii) we get that $\bar{R}$, hence also $R$, are $G$-quadratic.

Testing with Singular ([16]) the possible candidates for $J$, it is easy to check that all of them have a quadratic Gröbner basis with respect to revlex (usually induced by $\left.x_{2}>x_{3}>x_{4}>x_{5}\right)$. All of them possess a Koszul filtration and in all but one situation presented in Remark 14 there exists a Gröbner flag with basis $\left\{x_{2}, x_{3}, x_{4}, x_{5}\right\}$.

- If $h_{2}=3=\left(\begin{array}{l}3 \\ 2\end{array}\right)$, then $h_{3} \leqslant 1$ and $h_{4}=0$. Note that $J$ has at least two linearly independent squares of linear forms, namely $x_{2}^{2}$ and $x_{5}^{2}$. Under the assumption that $K$ is algebraically closed and of characteristic $\neq 2$, by Lemma 10(iv) we infer that $\bar{R}$, hence also $R$, is $G$-quadratic.

Scanning the possible candidates for $J$ by the method described above it turns out that there always exists a Koszul filtration for $\bar{R}$, without any restriction on the field $K$. In most cases this filtration is a Gröbner flag and the ideal $J$ has a quadratic Gröbner basis with respect to revlex (usually induced by $x_{2}>x_{3}>x_{4}>x_{5}$ ). There are though, up to a permutation of the variables, a couple of candidates for $J$ which do not admit a quadratic Gröbner basis with respect to any term order. We present these exceptions in Remark 15.

- If $h_{2}=4=\left(\begin{array}{l}3 \\ 2\end{array}\right)+\left(\begin{array}{l}1 \\ 1\end{array}\right)$, then $h_{3} \leqslant 1$ and $h_{4}=0$. We scanned the possible candidates for $J$ and we eliminated those ideals where the resolution of $K$ over $\bar{R}$ (computed with Singular [16]) is becoming nonlinear after at most 5 steps. All the other candidates had a quadratic Gröbner bases with respect to revlex (usually induced by $\left.x_{2}>x_{3}>x_{4}>x_{5}\right)$ and even a Gröbner flag. All the non-Koszul ideals were among those with $h_{3}=0$, hence with $e(H)=9$.

- If $h_{2}=5=\left(\begin{array}{l}3 \\ 2\end{array}\right)+\left(\begin{array}{l}2 \\ 1\end{array}\right)$, then by (2) we get $h_{3} \leqslant 2$ and $h_{4}=0$. It is well known and easy to see that if $\bar{R}$ is Koszul, then its Poincaré series equals $1 / H(-t)$. It is routine to check that if $h_{3}=0$ then $1 / H(-t)=1+4 t+\cdots-29 x^{6}+\cdots$, and if $h_{3}=1$ then $1 / H(-t)=1+4 t+\cdots-174 x^{8}+\cdots$. Therefore, in either case $R$ is not Koszul. However, if $h_{3}=2$ then $e(H)=12=2^{4}-2^{2}$ and we may apply Theorem 1 to conclude that $H$ is $G$-quadratic.

- If $h_{2}=6$, then $I_{H}^{*}$ is a complete intersection, hence $H$ is $G$-quadratic.

This finishes the proof of the theorem. 
Corollary 13. Let $H$ be a numerical semigroup with emb $\operatorname{dim}(H)=5$ and $e(H)$ different from 9, 10 and 11. Then $H$ is quadratic if and only if it is $G$-quadratic.

We next present the situations of Koszul ideals without Gröbner flags that occurred when discussing the cases $h_{2}=2$ and $h_{2}=3$ in the proof of Theorem 12 .

Remark 14. The ideal $J_{1}$ has a quadratic Gröbner basis with respect to revlex induced by $x_{2}>x_{3}>x_{4}>x_{5}$ and the $h$-vector of $\bar{R}=K\left[x_{2}, x_{3}, x_{4}, x_{5}\right] / J_{1}$ is $(1,4,2)$ :

$$
J_{1}=\left(x_{2}^{2}, x_{5}^{2}, p_{1}=x_{3}^{2}-x_{2} x_{4}, x_{4}^{2}, x_{2} x_{3}, x_{2} x_{5}, x_{3} x_{4}, x_{4} x_{5}\right) .
$$

The following computations show that

$$
\mathcal{J}_{1}=\left\{0,\left(x_{2}\right),\left(x_{2}, x_{3}\right),\left(x_{2}, x_{3}, x_{5}\right),\left(x_{2}, x_{4}, x_{5}\right),\left(x_{2}, x_{3}, x_{4}, x_{5}\right)\right\}
$$

is a Koszul filtration for $\bar{R}$ :

$$
\begin{array}{r}
0:\left(x_{2}\right)=\left(x_{2}, x_{3}, x_{5}\right), \quad\left(x_{2}\right):\left(x_{2}, x_{5}\right)=\left(x_{2}, x_{4}, x_{5}\right), \\
\left(x_{2}, x_{5}\right):\left(x_{2}, x_{3}, x_{5}\right)=\left(x_{2}, x_{5}\right):\left(x_{2}, x_{4}, x_{5}\right)=\left(x_{2}, x_{3}, x_{4}, x_{5}\right), \\
\left(x_{2}, x_{3}, x_{5}\right):\left(x_{2}, x_{3}, x_{4}, x_{5}\right)=\left(x_{2}, x_{4}, x_{5}\right):\left(x_{2}, x_{3}, x_{4}, x_{5}\right)=\left(x_{2}, x_{3}, x_{4}, x_{5}\right) .
\end{array}
$$

All the computations in these equations are made in $\bar{R}$. We hope there is no risk of confusion. It is also easy to check that there is no Gröbner flag for $\bar{R}$ with basis (the residue classes of) $x_{2}, x_{3}, x_{4}, x_{5}$.

Remark 15. The quotient of $\bar{S}=K\left[x_{2}, \ldots, x_{5}\right]$ modulo either one of the following two ideals has $h$-vector $(1,4,3)$ :

$$
\begin{aligned}
J_{2}=\left(x_{2}^{2}, x_{5}^{2}, p_{1}\right. & \left.=x_{3}^{2}-x_{2} x_{4}, p_{3}=x_{4}^{2}-x_{2} x_{5}, x_{2} x_{3}, x_{3} x_{4}, x_{3} x_{5}\right), \\
J_{3} & =\left(x_{2}^{2}, x_{5}^{2}, p_{1}=x_{3}^{2}-x_{2} x_{4}, x_{4}^{2}, x_{2} x_{3}, x_{3} x_{4}, x_{3} x_{5}\right) .
\end{aligned}
$$

We claim that none of them has a quadratic Gröbner basis with respect to any term order $<$.

Indeed, regarding $J_{2}$ : if $\operatorname{in}_{<}\left(p_{1}\right)=x_{2} x_{4}$ then the $S$-polynomial $S\left(p_{1}, x_{2} x_{3}\right)=x_{3}^{3}$. Else, in case $\operatorname{in}_{<}\left(p_{3}\right)=x_{4}^{2}$ we obtain $S\left(p_{1}, x_{3} x_{5}\right)=x_{2} x_{4} x_{5}$ and in case $\operatorname{in}_{<}\left(p_{3}\right)=x_{2} x_{5}$ we compute $S\left(p_{1}, x_{3} x_{4}\right)=x_{2} x_{4}^{2}$.

Regarding $J_{3}$ : if $\operatorname{in}_{<}\left(p_{1}\right)=x_{3}^{2}$, then $S\left(p_{1}, x_{3} x_{5}\right)=x_{2} x_{4} x_{5}$. Similarly, if in $\operatorname{in}_{<}\left(p_{1}\right)=x_{2} x_{4}$ then $S\left(p_{1}, x_{2} x_{3}\right)=x_{3}^{3}$. It is easy to observe that in any of these cases the computed $S$-polynomial does not reduce to zero using the remaining quadrics that generate $J_{2}$, respectively $J_{3}$. Therefore $J_{2}$ and $J_{3}$ do not have a quadratic Gröbner basis with respect to any term order.

The following computations performed in $\bar{R}=\bar{S} / J_{2}$, respectively in $\bar{R}=\bar{S} / J_{3}$, show that

$$
\mathcal{J}=\left\{0,\left(x_{5}\right),\left(x_{3}, x_{5}\right),\left(x_{2}, x_{5}\right),\left(x_{2}, x_{4}, x_{5}\right),\left(x_{2}, x_{3}, x_{5}\right),\left(x_{2}, x_{3}, x_{4}, x_{5}\right)\right\}
$$

is a Koszul filtration for $\bar{R}$ :

$$
\begin{array}{r}
0:\left(x_{5}\right)=\left(x_{3}, x_{5}\right),\left(x_{5}\right):\left(x_{3}, x_{5}\right)=\left(x_{2}, x_{4}, x_{5}\right),\left(x_{5}\right):\left(x_{2}, x_{5}\right)=\left(x_{2}, x_{3}, x_{5}\right) \\
\left(x_{3}, x_{5}\right):\left(x_{2}, x_{3}, x_{5}\right)=\left(x_{2}, x_{5}\right):\left(x_{2}, x_{4}, x_{5}\right)=\left(x_{2}, x_{3}, x_{4}, x_{5}\right), \\
\left(x_{2}, x_{3}, x_{5}\right):\left(x_{2}, x_{3}, x_{4}, x_{5}\right)=\left(x_{2}, x_{4}, x_{5}\right):\left(x_{2}, x_{3}, x_{4}, x_{5}\right)=\left(x_{2}, x_{3}, x_{4}, x_{5}\right) .
\end{array}
$$


Remark 16. In practice, we were not able to find quadratic numerical semigroups producing the ideals $J_{1}, J_{2}, J_{3}$ in Remarks 14 and 15. If such semigroups do not exist, we could drop the restrictions on the field $K$ in Theorem 12 .

Based on the proof of Theorem 12 and on the numerical experiments detailed before the proof, in Table 1 we summarize with examples our knowledge of the possible $h$-vectors of $\operatorname{gr}_{\mathfrak{m}} K[H]$, grouped by the multiplicity, when $H$ is a 5-generated quadratic numerical semigroup. For two of these $h$-vectors we could not find examples of semigroups, hence we ask if this list should be further reduced. The abbreviation quad GB indicates that $I_{H}^{*}$ has a quadratic Gröbner basis.

Table 1: $h$-vectors for quadratic 5-semigroups

\begin{tabular}{rlrr}
\hline \hline$e(H)$ & $h$-vector & Remarks on $I_{H}^{*}$ & Example \\
\hline 5 & $(1,4)$ & quad GB & $\langle 5,6,7,8,9\rangle$ \\
\hline 6 & $(1,4,1)$ & quad GB & $\langle 6,7,8,9,10\rangle$ \\
\hline 7 & $(1,4,2)$ & quad GB & $\langle 7,8,9,10,11\rangle$ \\
\hline 8 & $(1,4,2,1)$ & not CM, quad GB & $\langle 8,12,13,18,55\rangle$ \\
& $(1,4,3)$ & quad GB & $\langle 8,9,10,11,12\rangle$ \\
\hline 9 & $(1,4,3,1)$ & quad GB & $?$ \\
& $(1,4,4)$ & quad GB & $\langle 9,10,11,12,15\rangle$ \\
& & not Koszul & $\langle 9,17,20,23,25\rangle$ \\
\hline 10 & $(1,4,4,1)$ & quad GB & $\langle 10,16,19,22,25\rangle$ \\
& $(1,4,5)$ & not Koszul & $?$ \\
\hline 11 & $(1,4,5,1)$ & not Koszul & $\langle 11,13,14,15,19\rangle$ \\
\hline 12 & $(1,4,5,2)$ & quad GB & $\langle 12,14,16,18,27\rangle$ \\
\hline 16 & $(1,4,6,4,1)$ & quad GB & $\langle 16,17,18,20,24\rangle$ \\
& & &
\end{tabular}

Remark 17. In recent work ([11]) D'Anna et al. study the numerical semigroups $H$ for which the Hilbert function of $\operatorname{gr}_{\mathfrak{m}} K[H]$ is non-decreasing. We observe that this is also the case when $H$ is quadratic and $\operatorname{emb} \operatorname{dim}(H) \leqslant 5$.

Indeed, by Proposition 5 and the Table 1 we have that the $h$-vector of $\operatorname{gr}_{\mathfrak{m}} K[H]$ has nonnegative entries, hence the Hilbert function of $\operatorname{gr}_{\mathfrak{m}} K[H]$ is non-decreasing.

\section{A long proof}

In this section we prove the direct implication of Theorem 8.

Let $H$ be a quadratic numerical semigroup minimally generated by $a_{1}<\cdots<a_{5}$. Assume that $\operatorname{gr}_{\mathfrak{m}} K[H]$ is not Cohen-Macaulay.

By Theorem 1 we see that

$$
4<a_{1}<16 .
$$


By Proposition 4 and Lemma 3 the lack of the Cohen-Macaulay property is equivalent to say that there exist $\nu_{i} \in\{0,1\}, i=2, \ldots, 5$, such that $\sum_{i=2}^{5} \nu_{i} a_{i} \in a_{1}+H$ and whenever

$$
\sum_{i=2}^{5} \nu_{i} a_{i}=\sum_{i=1}^{5} \mu_{i} a_{i}, \text { with integers } \mu_{1}>0, \mu_{2}, \ldots, \mu_{5} \geqslant 0
$$

one has $\sum_{i=2}^{5} \nu_{i}>\sum_{i=1}^{5} \mu_{i}$.

Without loss of generality we may assume that in any equation like (4) one has $\nu_{i} \mu_{i}=0$ for all $i>1$. Since emb $\operatorname{dim}(H)=5$, at least two of the $\nu_{i}$ 's are positive. If exactly two of the $\nu_{i}$ 's are equal to 1 , then $\sum_{i=1}^{5} \mu_{i}=1, \mu_{1}=1$, and $a_{1} \in\left\langle a_{2}, \ldots, a_{5}\right\rangle$, which is false. If all $\nu_{i}$ are positive, then $a_{2}+\cdots+a_{5}=\mu_{1} a_{1}$ and since $a_{1}=e(H)$ we get $\mu_{1}>4=\sum_{i=2}^{5} \nu_{i}$, which contradicts the failure of the Cohen-Macaulay property.

Hence we have to consider only expressions where exactly one $\nu_{i}$ is zero. If $\nu_{2}=0$, then (4) is of the form $a_{3}+a_{4}+a_{5}=a_{1}+a_{2}$ or $a_{3}+a_{4}+a_{5}=2 a_{1}$. If $\nu_{3}=0$, then $a_{2}+a_{4}+a_{5}=a_{1}+a_{3}$ or $a_{2}+a_{4}+a_{5}=2 a_{1}$. If $\nu_{4}=0$, then $a_{2}+a_{3}+a_{5}=a_{1}+a_{4}$ or $a_{2}+a_{3}+a_{5}=2 a_{1}$. If $\nu_{5}=0$, then $a_{2}+a_{3}+a_{4}=a_{1}+a_{5}$ or $a_{2}+a_{3}+a_{4}=2 a_{1}$. Since $a_{1}<a_{2}<\cdots<a_{5}$, the only possibility for (4) is

$$
a_{2}+a_{3}+a_{4}=a_{1}+a_{5} .
$$

By Lemma 3(ii)

$$
\begin{aligned}
& 2 a_{2}=u a_{1}+v a_{3}+w a_{4}+\lambda a_{5}, \\
& 2 a_{3}=u^{\prime} a_{1}+v^{\prime} a_{2}+w^{\prime} a_{4}+\lambda^{\prime} a_{5}, \\
& 2 a_{4}=u^{\prime \prime} a_{1}+v^{\prime \prime} a_{2}+w^{\prime \prime} a_{3}+\lambda^{\prime \prime} a_{5},
\end{aligned}
$$

for $u, v, w, \lambda, u^{\prime}, \ldots, \lambda^{\prime \prime}$ nonnegative integers. Moreover, since all $a_{i}>0$ we may assume, without loss of generality, that $v, w, \lambda, v^{\prime}, w^{\prime}, \lambda^{\prime}, v^{\prime \prime}, w^{\prime \prime}, \lambda^{\prime \prime} \in\{0,1\}$. We later refer to these equations as normalized expressions for $2 a_{2}, 2 a_{3}$ and $2 a_{4}$, respectively.

We observe that due to the ordering of the $a_{i}$ 's and to (5) we have $a_{5}>a_{3}+a_{4}>$ $2 a_{3}>2 a_{2}$, hence $\lambda=\lambda^{\prime}=0$. Also, (5) implies $u^{\prime \prime} \lambda^{\prime \prime}=0$, otherwise $a_{4} \in\left\langle a_{1}, a_{2}, a_{3}\right\rangle$, which is false. Similarly, $v+w<2$.

The rest of the proof treats the remaining two possibilities: $2 a_{2}=u a_{1}+a_{3}$, or $2 a_{2}=$ $u a_{1}+a_{4}$, where we must have $u \geqslant 1$. The rather long discussion depends on the coefficients that occur in the normalized expressions (7) and (8). We identify six situations when the tangent cone $\mathrm{gr}_{\mathfrak{m}} K[H]$ is not Cohen-Macaulay, but, after reordering, all of them fit into the two families $(i)$ and $(i i)$ in the text of the theorem.

\subsection{Case (A)}

Assume

$$
2 a_{2}=u a_{1}+a_{3} \text { with } u \geqslant 1 \text {. }
$$




\subsubsection{Case $w^{\prime}=0$}

Then

$$
2 a_{3}=u^{\prime} a_{1}+v^{\prime} a_{2} \text { with } v^{\prime} \in\{0,1\} .
$$

If $v^{\prime}=1$, then $2 a_{3}=u^{\prime} a_{1}+a_{2}$, with $u^{\prime} \geqslant 1$. Adding to this the equation (9), after obvious simplifications we obtain $a_{2}+a_{3}=\left(u+u^{\prime}\right) a_{1}$, hence

$$
a_{5}=\left(a_{2}+a_{3}+a_{4}\right)-a_{1}=\left(u+u^{\prime}-1\right) a_{1}+a_{4} \in\left\langle a_{1}, a_{4}\right\rangle,
$$

which is false.

Thus $v^{\prime}=0$ and $2 a_{3}=u^{\prime} a_{1}$ where $u^{\prime}>1$ need to be odd and $a_{1}$ even. Together with (9) this gives $2\left(2 a_{2}-u a_{1}\right)=u^{\prime} a_{1}$, i.e.

$$
4 a_{2}=\left(u^{\prime}+2 u\right) \cdot a_{1} .
$$

Since $u^{\prime}$ is odd we get $4 \mid a_{1}$, hence $a_{1} \in\{8,12\}$.

1. If $\underline{a_{1}=8}$, then

$$
\begin{aligned}
& a_{2}=c\left(u^{\prime}+2 u\right), \\
& a_{3}=2 a_{2}-u a_{1}=4 u^{\prime} .
\end{aligned}
$$

Consider the normalized expression (8):

$$
2 a_{4}=u^{\prime \prime} a_{1}+v^{\prime \prime} a_{2}+w^{\prime \prime} a_{3}+\lambda^{\prime \prime} a_{5} .
$$

If $\lambda^{\prime \prime}=1$, since $a_{1}, a_{2}, a_{3}$ are even, then $a_{5}$ is even. Using (5) we infer that $a_{4}$ is even, which is false.

Therefore $\lambda^{\prime \prime}=0$ and

$$
2 a_{4}=u^{\prime \prime} a_{1}+v^{\prime \prime} a_{2}+w^{\prime \prime} a_{3} .
$$

Since $a_{2}, a_{3}<a_{4}$ we should have $u^{\prime \prime}>0$. Because $4 \mid a_{1}$ and $4 \mid a_{3}$, we can not have $v^{\prime \prime}=0$, otherwise $a_{4}$ is even, and by (5) also $a_{5}$ is even, which gives a contradiction. Hence $v^{\prime \prime}=1$. We distinguish two situations.

1.1. If $\underline{w}^{\prime \prime}=1$, then $2 a_{4}=u^{\prime \prime} a_{1}+a_{2}+a_{3}$. This gives

$$
\begin{aligned}
& a_{1}=8, \\
& a_{3}=4 u^{\prime}, \\
& a_{2}=4 u+2 u^{\prime}, \\
& a_{4}=4 u^{\prime \prime}+2 u+3 u^{\prime}, \\
& a_{5}=9 u^{\prime}+6 u+4 u^{\prime \prime}-8,
\end{aligned}
$$

which is of the desired form. 
1.2. If $\underline{w^{\prime \prime}=0}$, then $2 a_{4}=u^{\prime \prime} a_{1}+a_{2}$. This gives

$$
\begin{aligned}
& a_{1}=8, \\
& a_{3}=4 u^{\prime}, \\
& a_{2}=4 u+2 u^{\prime}, \\
& a_{4}=4 u^{\prime \prime}+2 u+u^{\prime}, \\
& a_{5}=7 u^{\prime}+6 u+4 u^{\prime \prime}-8,
\end{aligned}
$$

which is of the desired form.

2. If $a_{1}=12$, then

$$
\begin{aligned}
& a_{2}=3 u^{\prime}+6 u, \\
& a_{3}=6 u^{\prime} .
\end{aligned}
$$

In the normalized expression

$$
2 a_{4}=u^{\prime \prime} a_{1}+v^{\prime \prime} a_{2}+w^{\prime \prime} a_{3}+\lambda^{\prime \prime} a_{5}
$$

we add $a_{4}+\left(1-v^{\prime \prime}\right) a_{2}+\left(1-w^{\prime \prime}\right) a_{3}$ to both sides and using (5) we get

$$
3 a_{4}+\left(1-v^{\prime \prime}\right) a_{2}+\left(1-w^{\prime \prime}\right) a_{3}=\left(1+u^{\prime \prime}\right) a_{1}+\left(1+\lambda^{\prime \prime}\right) a_{5}
$$

hence $3 \mid a_{5}$, and by (5) also $3 \mid a_{4}$, hence $\operatorname{gcd}\left(a_{1}, \ldots, a_{5}\right)>1$, a contradiction.

\subsubsection{Case $w^{\prime}=1$}

Then

$$
2 a_{3}=u^{\prime} a_{1}+v^{\prime} a_{2}+a_{4} \text { with } v^{\prime} \in\{0,1\} .
$$

If $v^{\prime}=1$, then $2 a_{1}+2 a_{5}=2 a_{2}+2 a_{3}+2 a_{4}=\left(u a_{1}+a_{3}\right)+\left(u^{\prime} a_{1}+a_{2}+a_{4}\right)+2 a_{4}=$ $\left(u^{\prime}+u\right) a_{1}+\left(a_{2}+a_{3}+a_{4}\right)+2 a_{4}$. This gives $a_{5}=\left(u^{\prime}+u-1\right) a_{1}+2 a_{4}$, which is false. Therefore $v^{\prime}=0$ and

$$
2 a_{3}=u^{\prime} a_{1}+a_{4} \text { with } u^{\prime}>0 .
$$

Consider the normalized expression (8)

$$
2 a_{4}=u^{\prime \prime} a_{1}+v^{\prime \prime} a_{2}+w^{\prime \prime} a_{3}+\lambda^{\prime \prime} a_{5} .
$$

If $\lambda^{\prime \prime}=1$, since $u^{\prime \prime} \lambda^{\prime \prime}=0$, we get $u^{\prime \prime}=0$. Equation (8) becomes

$$
2 a_{4}=v^{\prime \prime} a_{2}+w^{\prime \prime} a_{3}+a_{5} .
$$

To this we add (13) and $2 a_{2}=u a_{1}+a_{3}$ from (9) and after using (5) we get that

$$
a_{5}=\left(u^{\prime}+u-2\right) a_{1}+v^{\prime \prime} a_{2}+\left(w^{\prime \prime}+1\right) a_{3}+a_{4} \in\left\langle a_{1}, a_{2}, a_{3}, a_{4}\right\rangle,
$$

which is false. 
Thus $\lambda^{\prime \prime}=0$ and $2 a_{4}=u^{\prime \prime} a_{1}+v^{\prime \prime} a_{2}+w^{\prime \prime} a_{3}$. To this we add (13) and $2 a_{2}=u a_{1}+a_{3}$ and, after using (5), we see that

$$
2 a_{5}=\left(u^{\prime \prime}+u^{\prime}+u-2\right) a_{1}+v^{\prime \prime} a_{2}+\left(w^{\prime \prime}+1\right) a_{3}+a_{4} .
$$

If $v^{\prime \prime}>0$, by (5) we get $a_{5} \in\left\langle a_{1}, a_{2}, a_{3}, a_{4}\right\rangle$, which is false.

Hence $v^{\prime \prime}=0$ and (8) becomes

$$
2 a_{4}=u^{\prime \prime} a_{1}+w^{\prime \prime} a_{3}=u^{\prime \prime} a_{1}+w^{\prime \prime}\left(2 a_{2}-u a_{1}\right)=\left(u^{\prime \prime}-w^{\prime \prime} u\right) a_{1}+2 w^{\prime \prime} a_{2} \text { with } w^{\prime \prime} \in\{0,1\} .
$$

From (13) we extract

$$
a_{4}=2 a_{3}-u^{\prime} a_{1}=2\left(2 a_{2}-u a_{1}\right)-u^{\prime} a_{1}=4 a_{2}-\left(2 u+u^{\prime}\right) a_{1}
$$

which we replace in the previous equation for $2 a_{4}$. Routine manipulation gives

$$
\left(8-2 w^{\prime \prime}\right) a_{2}=\left(\left(4-w^{\prime \prime}\right) u+2 u^{\prime}+u^{\prime \prime}\right) a_{1} .
$$

1. If $\underline{w^{\prime \prime}=0}$, the equations (14), (9) and (8) together with (5) yield

$$
\begin{aligned}
& a_{2}=\frac{4 u+2 u^{\prime}+u^{\prime \prime}}{8} \cdot a_{1}, \\
& a_{3}=\frac{2 u^{\prime}+u^{\prime \prime}}{4} \cdot a_{1}, \\
& a_{4}=\frac{u^{\prime \prime}}{2} \cdot a_{1}, \\
& a_{5}=\frac{4 u+6 u^{\prime}+7 u^{\prime \prime}-8}{8} \cdot a_{1} .
\end{aligned}
$$

From here we infer that $u^{\prime \prime}$ is odd (otherwise $a_{1}$ divides $a_{4}$, which is false) and consequently $8 \mid a_{1}$. Hence $a_{1}=8$ and the generators of the semigroup are

$$
\begin{aligned}
& a_{1}=8, \\
& a_{4}=4 u^{\prime \prime}, \\
& a_{3}=2 u^{\prime \prime}+4 u^{\prime}, \\
& a_{2}=u^{\prime \prime}+2 u^{\prime}+4 u, \\
& a_{5}=4 u+6 u^{\prime}+7 u^{\prime \prime}-8,
\end{aligned}
$$

as desired.

2. If $\underline{w^{\prime \prime}=1}$, equation (14) together with (9), (8) and (5) give after routine computations

$$
\begin{aligned}
& a_{2}=\frac{3 u+2 u^{\prime}+u^{\prime \prime}}{6} \cdot a_{1}, \\
& a_{3}=\frac{2 u^{\prime}+u^{\prime \prime}}{3} \cdot a_{1}, \\
& a_{4}=\frac{u^{\prime}+2 u^{\prime \prime}}{3} \cdot a_{1}, \\
& a_{5}=\frac{3 u+8 u^{\prime}+7 u^{\prime \prime}-6}{6} \cdot a_{1} .
\end{aligned}
$$

We note that $a_{5}=a_{2}+\left(u^{\prime}+u^{\prime \prime}-1\right) a_{1} \in\left\langle a_{1}, a_{2}\right\rangle$, which is false. 


\subsection{Case (B)}

Assume

$$
2 a_{2}=u a_{1}+a_{4}, \text { with } u \geqslant 1 .
$$

If in the normalized expression

$$
2 a_{4}=u^{\prime \prime} a_{1}+v^{\prime \prime} a_{2}+w^{\prime \prime} a_{3}+\lambda^{\prime \prime} a_{5} \quad \text { with } v^{\prime \prime}, w^{\prime \prime}, \lambda^{\prime \prime} \in\{0,1\}
$$

we had $\lambda^{\prime \prime}=1$, then $u^{\prime \prime}=0$. Combined with (16), we get

$$
2 a_{2}+a_{4}=u a_{1}+2 a_{4}=u a_{1}+v^{\prime \prime} a_{2}+w^{\prime \prime} a_{3}+a_{5} .
$$

Using (5) and the latter equation we obtain

$$
\left(1-v^{\prime \prime}\right) a_{2}=(u-1) a_{1}+\left(w^{\prime \prime}+1\right) a_{3}>0,
$$

which is a contradiction since $v^{\prime \prime} \leqslant 1$. Therefore $\lambda^{\prime \prime}=0$.

\subsubsection{Case $w^{\prime}=v^{\prime}=0$}

Then $u^{\prime}>0$ and (7) becomes

$$
2 a_{3}=u^{\prime} a_{1} .
$$

From this and (16) we get $a_{3}=u^{\prime} a_{1} / 2, a_{4}=2 a_{2}-u a_{1}$ and that $a_{1}$ is even and $u^{\prime}$ is odd.

We substitute in the normalized expression above the values for $a_{3}$ and $a_{4}$ in terms of $a_{1}$ and $a_{2}$ and we get

$$
\left(4-v^{\prime \prime}\right) \cdot a_{2}=\left(2 u+u^{\prime \prime}+w^{\prime \prime} \frac{u^{\prime}}{2}\right) \cdot a_{1}
$$

1. If $\underline{v^{\prime \prime}=1}$, the previous equation becomes

$$
3 a_{2}=\left(2 u+u^{\prime \prime}+w^{\prime \prime} \frac{u^{\prime}}{2}\right) \cdot a_{1} .
$$

We consider the possible even values of $a_{1}$.

1.1. If $a_{1}=6$ we obtain the other generators

$$
\begin{aligned}
& a_{2}=4 u+2 u^{\prime \prime}+w^{\prime \prime} u^{\prime}, \\
& a_{3}=3 u^{\prime}, \\
& a_{4}=2 u+4 u^{\prime \prime}+2 w^{\prime \prime} u^{\prime}, \\
& a_{5}=6 u+6 u^{\prime \prime}+\left(3 w^{\prime \prime}+3\right) u^{\prime}-6 .
\end{aligned}
$$

Note that $a_{5}=\left(u+u^{\prime \prime}-1\right) a_{1}+\left(w^{\prime \prime}+1\right) a_{3} \in\left\langle a_{1}, a_{3}\right\rangle$, which is false.

1.2. If $a_{1}=8$ or 12 , then $a_{2}, a_{3}, a_{4}$ are even, hence $a_{5}$ is even as well, a contradiction.

1.3. If $\underline{a_{1}=10}$, then it easy to see that $a_{2}, a_{3}, a_{4}, a_{5}$ are divisible by 5 , which is false. 
1.4. If $a_{1}=14$, then all the generators are divisible by 7 , which is false.

2. If $\underline{v^{\prime \prime}=0}$, then

$$
4 a_{2}=\left(2 u+u^{\prime \prime}+w^{\prime \prime} \frac{u^{\prime}}{2}\right) \cdot a_{1},
$$

which forces $a_{1}$ to be even.

2.1. If $\underline{w^{\prime \prime}=0}$ we get $2 a_{4}=u^{\prime \prime} a_{1}$ and $4 a_{2}=\left(2 u+u^{\prime \prime}\right) a_{1}$. Therefore $u^{\prime \prime}$ is odd, $a_{1}$ is divisible by 4 , hence $a_{1} \in\{4,8\}$, and the other generators are

$$
\begin{aligned}
& a_{2}=\frac{2 u+u^{\prime \prime}}{4} \cdot a_{1}, \\
& a_{3}=\frac{u^{\prime}}{2} \cdot a_{1}, \\
& a_{5}=\frac{3 u^{\prime \prime}+2 u+2 u^{\prime}-4}{4} \cdot a_{1} .
\end{aligned}
$$

It is immediate to note that if $a_{1}=8$ all generators are even, while if $a_{1}=12$ all of them are divisible by 3 . None of these situations may hold.

2.2. If $\underline{w^{\prime \prime}=1}$ we obtain

$$
8 a_{2}=\left(4 u+2 u^{\prime \prime}+u^{\prime}\right) \cdot a_{1} .
$$

Since $u^{\prime}$ is odd we get $a_{1}=8$. From the other equations we compute the other generators

$$
\begin{aligned}
& a_{3}=4 u^{\prime}, \\
& a_{4}=4 u^{\prime \prime}+2 u^{\prime}, \\
& a_{2}=4 u+2 u^{\prime \prime}+u^{\prime}, \\
& a_{5}=4 u+6 u^{\prime \prime}+7 u^{\prime}-8,
\end{aligned}
$$

which turn out to be of the desired form.

\subsubsection{Case $w^{\prime}=0$ and $v^{\prime}=1$}

Then $2 a_{3}=u^{\prime} a_{1}+a_{2}$ with $u^{\prime}>0$.

Using (16) and the normalized equation (8) we get

$$
2 a_{1}+2 a_{5}=2 a_{2}+2 a_{3}+2 a_{4}=\left(u^{\prime}+u^{\prime \prime}+u\right) a_{1}+\left(v^{\prime \prime}+1\right) a_{2}+w^{\prime \prime} a_{3}+a_{4},
$$

hence $w^{\prime \prime}=0$.

1. If $\underline{v^{\prime \prime}=1}$, by adding the equations $2 a_{2}=u a_{1}+a_{4}$ and $2 a_{4}=u^{\prime \prime} a_{1}+a_{2}$ we get $a_{2}+a_{4}=$ $\left(u+u^{\prime \prime}\right) a_{1}$. Therefore $a_{5}=\left(a_{2}+a_{4}\right)+a_{3}-a_{1}=\left(u+u^{\prime \prime}-1\right) a_{1}+a_{3}$, which is false. 
2. If $\underline{v^{\prime \prime}=0}$, then $2 a_{4}=u^{\prime \prime} a_{1}$ with $u^{\prime \prime}$ odd and $a_{1}$ even. Since $2 a_{2}=u a_{1}+a_{4}$ we get $4 a_{2}=\left(2 u+u^{\prime \prime}\right) \cdot a_{1}$. This implies that $4 \mid a_{1}$, hence $a_{1} \in\{8,12\}$.

If $\underline{a_{1}=12}$, then $a_{2}=3\left(2 u+u^{\prime \prime}\right)$. Since $2 a_{3}=u^{\prime} a_{1}+a_{2}$ and $a_{4}=u a_{1}-2 a_{2}$ we derive that $a_{3}$ and $a_{4}$ are divisible by 3 , hence also $3 \mid a_{5}$, which is false.

If $\underline{a_{1}=8}$, the rest of the generators are

$$
\begin{aligned}
& a_{4}=4 u^{\prime \prime}, \\
& a_{2}=4 u+2 u^{\prime \prime}, \\
& a_{3}=2 u+u^{\prime \prime}+4 u^{\prime}, \\
& a_{5}=6 u+7 u^{\prime \prime}+4 u^{\prime}-8,
\end{aligned}
$$

which are of the desired format.

\subsubsection{Case $w^{\prime}=1$}

Then

$$
2 a_{3}=u^{\prime} a_{1}+v^{\prime} a_{2}+a_{4}
$$

Since in the normalized expression (8) we have $\lambda^{\prime \prime}=0$ and $v^{\prime \prime}, w^{\prime \prime} \leqslant 1$, then $u^{\prime \prime}>0$. By

(16) and (20) we may write

$$
\begin{aligned}
2 a_{5} & =2 a_{2}+2 a_{3}+2 a_{4}-2 a_{1} \\
& =\left(u a_{1}+a_{4}\right)+\left(u^{\prime} a_{1}+v^{\prime} a_{2}+a_{4}\right)+\left(u^{\prime \prime} a_{1}+v^{\prime \prime} a_{2}+w^{\prime \prime} a_{3}\right)-2 a_{1} \\
& =\left(u+u^{\prime}+u^{\prime \prime}-2\right) a_{1}+\left(v^{\prime}+v^{\prime \prime}\right) a_{2}+w^{\prime \prime} a_{3}+2 a_{4} .
\end{aligned}
$$

1. If $\underline{w^{\prime \prime}=1 \text { and } v^{\prime}+v^{\prime \prime}>0}$, we get $a_{5} \in\left\langle a_{1}, a_{2}, a_{3}, a_{4}\right\rangle$, which is false.

2. If $\underline{w^{\prime \prime}=1 \text { and } v^{\prime}=v^{\prime \prime}=0}$, summing the equations

$$
\begin{aligned}
& 2 a_{4}=u^{\prime \prime} a_{1}+a_{3} \\
& 2 a_{3}=u^{\prime} a_{1}+a_{4}
\end{aligned}
$$

we obtain that $a_{3}+a_{4}=\left(u^{\prime}+u^{\prime \prime}\right) a_{1}$, which, together with (5) yields $a_{5}=\left(u^{\prime}+u^{\prime \prime}-\right.$ 1) $a_{1}+a_{2} \in\left\langle a_{1}, a_{2}\right\rangle$, a contradiction.

3. If $\underline{w^{\prime \prime}=0}$, then $2 a_{4}=u^{\prime \prime} a_{1}+v^{\prime \prime} a_{2}$, and after substituting in here $a_{4}=2 a_{2}-u a_{1}$ (from (16)) we get

$$
\left(4-v^{\prime \prime}\right) a_{2}=\left(2 u+u^{\prime \prime}\right) a_{1} .
$$

3.1. If $\underline{v^{\prime \prime}=0}$, then $a_{4}=u^{\prime \prime} a_{1} / 2$ and $u^{\prime \prime}$ is odd. Other generators are obtained immediately:

$$
\begin{aligned}
& a_{2}=\frac{2 u+u^{\prime \prime}}{4} \cdot a_{1}, \\
& a_{3}=\frac{4 u^{\prime}+2 u^{\prime \prime}+v^{\prime}\left(2 u+u^{\prime \prime}\right)}{8} \cdot a_{1} .
\end{aligned}
$$


Since $u^{\prime \prime}$ is odd we deduce that $4 \mid a_{1}$, hence $a_{1} \in\{8,12\}$.

If $a_{1}=12$, because the denominators of $a_{2}, a_{3}$ and $a_{4}$ are powers of 2 we see that $a_{2}, a_{3}, a_{4}$ are divisible by 3 , and the same holds for $a_{5}$, a contradiction.

Assume $a_{1}=8$. If $v^{\prime}=0$ then we note that $a_{2}, a_{3}, a_{4}$ are even, hence $a_{5}$ is even, too. This is false. Hence $v^{\prime}=1$, and the generators of the semigroup are

$$
\begin{aligned}
& a_{1}=8, \\
& a_{4}=4 u^{\prime \prime}, \\
& a_{2}=4 u+2 u^{\prime \prime}, \\
& a_{3}=4 u^{\prime}+2 u+3 u^{\prime \prime}, \\
& a_{5}=4 u^{\prime}+6 u+9 u^{\prime \prime}-8,
\end{aligned}
$$

which is on our list.

3.2. If $\underline{v^{\prime \prime}=1}$, then $3 a_{2}=\left(2 u+u^{\prime \prime}\right) a_{1}$ which implies $3 \nmid 2 u+u^{\prime \prime}$ and $3 \mid a_{1}$. We get

$$
a_{2}=\frac{2 u+u^{\prime \prime}}{3} \cdot a_{1}, \quad a_{4}=2 a_{2}-u=\frac{2 u^{\prime \prime}+u}{3} \cdot a_{1} .
$$

By (5) we see that $a_{5}=a_{2}+a_{3}+a_{4}-a_{1}=\left(u+u^{\prime \prime}-1\right) a_{1}+a_{3}$, which is false.

The proof of Theorem 8 is now complete.

\section{Acknowledgements}

We gratefully acknowledge the use of the computer algebra system Singular [16] for our numerical experiments. We thank Aldo Conca for pointing our attention to [6]. We thank Mihai Cipu for verifying the proof of Theorem 8 and the suggested improvements.

\section{References}

[1] J. Backelin, A distributiveness property of augmented algebras and some related homological results, Ph.D. Thesis, Stockholm University, 1982.

[2] J. Backelin, R. Fröberg, Poincaré Series of Short Artinian Rings, J. Algebra 96 (1985), 495-498.

[3] V. Barucci, R. Fröberg, Associated graded rings of one-dimensional analytically irreducible rings, J. Algebra 304 (2006), 349-358.

[4] A. Conca, Gröbner Bases for Spaces of Quadrics of Low Codimension, Advances in Applied Mathematics 24 (2000), 111-124.

[5] A. Conca, M.E. Rossi, G. Valla, Gröbner Flags and Gorenstein Algebras, Compositio Mathematica 129 (2001), 95-121.

[6] A. Conca, Gröbner bases for spaces of quadrics of codimension 3, J. Pure and Applied Algebra 213 (2009), 1564-1568. 
[7] A. Conca, E. De Negri, M.E. Rossi, Koszul algebras and regularity, Commutative algebra, 285-315, Springer, New York, 2013.

[8] A. Conca, N.V. Trung, G. Valla, Koszul property for points in projective spaces, Math. Scand. 89 (2001) no. 2, 201-216.

[9] A. D'Alì, The Koszul property for spaces of quadrics of codimension three, 2016, preprint 26 pp. arXiv:1605.09145.

[10] M. D'Anna, V. Micale, A. Sammartano, When the associated graded ring of a semigroup ring is Complete Intersection, J. Pure and Applied Algebra 217 (2013), 10071017.

[11] M. D'Anna, M. Di Marca, V. Micale, On the Hilbert function of the tangent cone of a monomial curve, Semigroup Forum 91 (2015), 718-730.

[12] C. Delorme, Sous-monö̈des d'intersection complète de N, Ann. Sci. Ecole Norm. Sup. (4) 9 (1976), no. 1, 145-154.

[13] D. Eisenbud, Commutative Algebra with a View Toward Algebraic Geometry, Graduate Texts in Mathematics 150, Springer, 1995.

[14] D. Eisenbud, M. Green, J. Harris, Higher Castelnuovo Theory, Asterisque 218 (1993), $187-202$.

[15] V. Ene, J. Herzog, Gröbner bases in commutative algebra, Graduate Studies in Mathematics 130, American Mathematical Society, 2012.

[16] W. Decker, G.-M. Greuel, G. Pfister, H. Schönemann, Singular 3-1-6 - A computer algebra system for polynomial computations. http://www. singular. uni-kl.de (2012).

[17] A. Garcia, Cohen-Macaulayness of the associated graded of a semigroup ring, Communications in Algebra 10 (1982), no. 4, 393-415.

[18] J. Herzog, When is a regular sequence super regular?, Nagoya Math. J. 83 (1981), $183-195$.

[19] J. Herzog, D.I. Stamate, Quadratic numerical semigroups and the Koszul property, Kyoto Journal of Mathematics, in press, arXiv:1510.00935.

[20] A. Onetto, F. Strazzanti, G. Tamone, One-dimensional Gorenstein local rings with decreasing Hilbert function, 2016, preprint 21 pp. arXiv:1602.00334.

[21] V. Reiner, D.I. Stamate, Koszul incidence algebras, affine semigroups, and StanleyReisner ideals, Adv. Math. 224 (2010), no. 6, 2312-2345.

[22] L. Robbiano, G. Valla, On the equations defining tangent cones, Math. Proc. Camb. Phil. Soc. 88 (1980), 281-297.

[23] M.E. Rossi, G. Valla, Multiplicity and t-isomultiple ideals, Nagoya Math. J. 110 (1988), 81-111.

[24] J.E. Roos, B. Sturmfels, A toric ring with irrational Poincaré-Betti series, C. R. Acad. Sci. Paris, 326 (1998), Serie I, 141-146.

[25] J.D. Sally, On the associated graded ring of a local Cohen-Macaulay ring, J. Math. Kyoto Univ. 17 (1977), no. 1, 19-21. 